\title{
Filovirus vaccines
}

\section{Steven B. Bradfute, John M. Dye and Sina Bavari*}

United States Army Medical Research Institute of Infectious Diseases; National Interagency Biodefense Campus; Fort Detrick; Frederick, MD USA

Key words: ebola, ebola virus, ebolavirus, filovirid, filoviridae, filovirus, marburg virus, marburgvirus, mononegavirad, mononegavirales, mononegavirus, vaccine

Filoviruses can cause severe and often fatal hemorrhagic fever in humans and non-human primates (NHPs). Although there are currently no clinically proven treatments for filovirus disease, much progress has been made in recent years in the discovery of therapeutics and vaccines against these viruses. A variety of vaccine platforms have been shown to be effective against filovirus infection. This review summarizes the literature in this field, focusing on vaccines that have been shown to protect NHPs from infection. Furthermore, the uses of rodent models in vaccine development, as well as correlates of immunity, are discussed.

\section{Introduction}

The filoviruses-marburgviruses, ebolaviruses and cuevaviruses - are enveloped, negative strand RNA viruses, and many of them cause often fatal severe hemorrhagic fever in humans and non-human primates (NHP). Filoviruses have seven genes: glycoprotein (GP), nucleoprotein (NP), RNA-dependent RNA polymerase (L), matrix protein (VP40), polymerase cofactor (VP35), transcriptional activator (VP30) and secondary matrix protein (VP24). ${ }^{1}$ The disease course is characterized by fever, elevated serum levels of aspartate aminotransferase (AST) and/or alanine transaminase (ALT), malaise, myalgia, coagulopathy and high lethality rates (reviewed in ref. 2). Five ebolaviruses have been described-Ebola (EBOV, previously referred to as Zaire), Reston (RESTV), Bundibugyo (BDBV), Sudan (SUDV) and Taï Forest (TAFV, previously Ivory Coast). RESTV is the only ebolavirus that is not known to be pathogenic in humans. There are two marburgviruses, Marburg (MARV) and Ravn (RAVV) and one proposed "cuevavirus," Lloviu (LLOV). ${ }^{3}$ Several MARV variants are used in research, including Musoke, Cieplik (formerly known as $\mathrm{Ci67)}$ and Angola. The published literature suggests that animals protected against one marburgvirus are subsequently protected against other marburgviruses. ${ }^{3}$ There appears to be more variability in cross-protection with ebolaviruses; EBOV and SUDV infection appears to generate only moderate cross-protective responses, whereas EBOV and SUDV dual vaccination may protect against BDBV infection (discussed below). LLOV was recently discovered in bats and its pathogenicity in humans is unknown.

*Correspondence to: Sina Bavari; Email: sina.bavari@us.army.mil Submitted: 12/20/10; Revised: 02/18/11; Accepted: 02/22/11

DOI: $10.4161 /$ hv.7.6.15398
Filoviruses are NIAID Category A Priority Pathogens because of their high lethality in humans (ranging from 23-90\%, depending on the virus and location of outbreak), ability to be infectious by the aerosol route and concerns over their possible use as bioweapons. ${ }^{4-7}$ Filoviruses are endemic in parts of Africa (MARV, RAVV, BDBV, EBOV, SUDV and TAFB), the Philippines (REBOV) and possibly Europe (LLOV). Most ebolavirus outbreaks tend to occur in central and western African rain forests, while marburgvirus infections are more prevalent in arid woodlands of western, south-central and eastern Africa. ${ }^{1}$ MARV is thought to circulate in bat populations and there is suggestive evidence that bats are also carriers for EBOV and LLOV., ${ }^{3,8-11}$ The viruses are then thought to be transferred from bats to NHPs and humans, in which infection is acute and pathogenic. However, it has recently been reported that domestic swine in the Philippines are hosts for REBOV ${ }^{12}$ suggesting that other animals may act as carriers for these viruses.

Several animal models are available for the study of filovirus disease (Table 1). Multiple species of NHPs, including rhesus monkeys, cynomolgus macaques, African green monkeys, vervet monkeys and baboons, are susceptible to wild-type filovirus infection. However, it should be noted that there are substantial differences in filoviral pathogenesis and host response to infection in these different NHPs. ${ }^{13-16}$ Rhesus monkeys and cynomolgus macaques are most frequently used as NHP models and, based on very limited human clinical data, are thought to more accurately model filovirus disease in humans. Guinea pig and laboratory mouse models are also available for the study of filoviruses. Due to expense, space and assay limitations of NHP models, the use of rodent models for filovirus studies are indispensible for early testing and modification of vaccines and therapeutics, as well as determining correlates of immunity. The available data suggest that wild-type filoviruses do not cause complete lethality in adult, immocompetent laboratory mice or guinea pigs, but serial passage of the virus through the rodent of interest has produced adapted filovirus strains that are lethal in these models. ${ }^{17-21}$ Although rodent models are not always predictive of efficacy in NHPs, it is important to note that all of the vaccines discussed here, which are effective in NHPs, have been shown to be effective in at least one rodent model prior to testing in NHPs. The usefulness of these models can be shown in early-stage vaccine studies conducted in laboratory mice to determine dosage and adjuvant requirements. ${ }^{22-24}$ Questions regarding mechanisms of immunity for various vaccine platforms have been examined using knockout mice and adoptive cell transfer assays, ${ }^{22,24-26}$ which are difficult or impossible to do in NHP models. 
Table 1. Lethal laboratory animal models for filovirus infection

\begin{tabular}{|c|c|c|c|c|c|c|c|}
\hline Virus & $\begin{array}{l}\text { Suckling } \\
\text { mice }\end{array}$ & $\begin{array}{l}\text { Adult } \\
\text { mice }\end{array}$ & $\begin{array}{c}\text { Immunodeficient } \\
\text { mice }\end{array}$ & $\begin{array}{l}\text { Rhesus } \\
\text { macaque }\end{array}$ & $\begin{array}{l}\text { Cynomolgus } \\
\text { macaque }\end{array}$ & Guinea pig & $\begin{array}{c}\text { Rodent-adapted } \\
\text { variants }\end{array}$ \\
\hline EBOV & Yes $^{79-82}$ & $\mathrm{No}^{83}$ & Yes $^{83}$ & Yes $^{14,15}$ & Yes $^{84}$ & $\mathrm{No}^{19,82,85}$ & Guinea pig, ${ }^{19,85}$ mouse $^{18}$ \\
\hline SUDV & ND & $\mathrm{No}^{83}$ & Yes $^{83}$ & Partial ${ }^{43,65,85}$ & Yes $^{84}$ & $\mathrm{No}^{82,85}$ & Guinea pig ${ }^{86}$ \\
\hline TAFV & ND & $\mathrm{No}^{83}$ & $\mathrm{No}^{83}$ & ND & Partial ${ }^{60}$ & ND & ND \\
\hline BDBV & ND & ND & ND & ND & Partial $^{52}$ & ND & ND \\
\hline RESTV & ND & $\mathrm{No}^{83}$ & $\mathrm{No}^{83}$ & $Y_{e s}^{87}$ & Partial ${ }^{84,88}$ & ND & ND \\
\hline MARV & Yes $^{89}$ & $\mathrm{No}^{83}$ & Yes $^{83,90}$ & Yes $^{91}$ & Yes $^{31}$ & $\mathrm{No}^{17,20}$ & Guinea pig 17,20 \\
\hline RAVV & ND & $\mathrm{No}^{83}$ & $Y_{e s}^{21,83}$ & Yes $^{92}$ & Yes $^{93}$ & $\mathrm{No}^{20}$ & Guinea pig, ${ }^{94}$ Mouse $^{21}$ \\
\hline
\end{tabular}

ND, Not determined; Yes, lethal; no, not lethal. All rodent-adapted varients listed are lethal.

Table 2. Summary of filovirus vaccines that are efficacious in non-human primates

\begin{tabular}{|c|c|c|c|c|c|c|}
\hline Platform & $\begin{array}{c}\text { Genes/proteins in } \\
\text { vaccine }\end{array}$ & $\begin{array}{l}\text { Viruses } \\
\text { protected } \\
\text { against }\end{array}$ & Antibody titers* & $\begin{array}{l}\text { Neutralizing } \\
\text { antibody titers }\end{array}$ & T-cell responses & References \\
\hline $\begin{array}{c}\text { Vesicular stomatitis } \\
\text { virus (VSV) }\end{array}$ & GP & $\begin{array}{c}\text { MARV, RAVV, } \\
\text { EBOV, SUDV, TAFV }\end{array}$ & Low to moderate & Undetectable to low & None detected & $58,60,93$ \\
\hline $\begin{array}{l}\text { VEE replicon particles } \\
\text { (VRP) }\end{array}$ & $\begin{array}{l}\text { GP, GP+NP (NP alone } \\
\text { partially protective) }\end{array}$ & MARV & Low to moderate & None & Not tested & 31 \\
\hline Adenovirus (AdV) & $\mathrm{GP}, \mathrm{GP}+\mathrm{NP}$ & $\begin{array}{l}\text { MARV, EBOV, } \\
\text { SUDV }\end{array}$ & $\begin{array}{l}\text { Moderate to } \\
\text { High }\end{array}$ & Undetectable to low & $\mathrm{CD}^{+}$and $\mathrm{CD}^{+}$ & $\begin{array}{c}39,40,42 \\
49,95\end{array}$ \\
\hline DNA & GP & MARV & Moderate & Undetectable to low & $\mathrm{CD}^{+}$and $\mathrm{CD}^{+}$ & 49,50 \\
\hline $\begin{array}{l}\text { DNA prime-AdV } \\
\text { boost }\end{array}$ & $\begin{array}{l}\text { GP+NP (EBOV); GP } \\
\text { (MARV, BDBV); }\end{array}$ & $\begin{array}{l}\text { MARV, EBOV, } \\
\text { BDBV }\end{array}$ & $\begin{array}{l}\text { Moderate to high } \\
\text { (EBOV, MARV); } \\
\text { none (BDBV) }\end{array}$ & $\begin{array}{l}\text { Undetectable to low } \\
\text { (EBOV, MARV) }\end{array}$ & $\begin{array}{c}\mathrm{CD}^{+} \text {and } \mathrm{CD}^{+} \\
(\mathrm{BDBV}, \mathrm{EBOV} \\
\text { MARV) }\end{array}$ & $38,39,49,52$ \\
\hline Virus-like particles & $G P+N P+V P 40$ & $\begin{array}{l}\text { MARV, RAVV, } \\
\text { EBOV }\end{array}$ & Moderate to high & $\begin{array}{l}\text { Low to moderate } \\
\text { (EBOV) }\end{array}$ & Yes (EBOV) & 74,96 \\
\hline $\begin{array}{l}\text { Human parainfluenza } \\
\text { virus type } 3 \text { (HPIV-3) }\end{array}$ & $\mathrm{GP}, \mathrm{GP}+\mathrm{NP}$ & EBOV & Low to moderate & NT & $\begin{array}{l}\text { Low to unde- } \\
\text { tectable }\end{array}$ & 70 \\
\hline
\end{tabular}

*For this review, low antibody titers are defined as 300-5,000; moderate as 5,000-50,000; and high as $~ 50,000$ or above. Antibody titers are based on individual publications, often using different methods, and cannot be assumed to be comparable across platforms.

Initial attempts to generate filovirus vaccines focused on the use of inactivated whole virions and had mixed success in NHP models. ${ }^{27-29}$ Later studies began to test virus-like particles, viral vectors or plasmid DNA expressing filovirus genes as vaccine platforms. Most platforms were based on generating immune responses against GP, with the hope of generating neutralizing antibody that would protect against filovirus infection. Even now, however, it is unclear what types of immune responses need to be induced for successful vaccination against filovirus infection. To date, several different vaccine platforms have protected NHPs from marburgvirus and/or ebolavirus challenge (summarized in Table 2). This broad array of protective vaccine platforms suggests that there are multiple ways to abrogate filovirus pathogenesis. For example, as discussed below, anti-filoviral antibody titers are good correlates of immunity in some vaccine platforms, but not in others. Even within the same platform, mechanisms of immunity may differ based on the filovirus being tested.

Despite increased interest in studying these viruses, there are many aspects of filovirus pathogenesis and immunity that remain unknown. Due to their high lethality rates, research on filoviruses is confined to maximum-containment laboratories.
Filovirus infections in humans tend to be rare and sporadic, so there is a paucity of samples from infected patients available for research. Additionally, there is a bevy of data that is reported at meetings and informally discussed amongst filovirologists, but is not peer reviewed or published. As a result of these limitations, information concerning vaccination, therapeutics and immune responses to filovirus infection is incomplete.

This review focuses on the efficacy and correlates of immunity of filovirus vaccine studies in animal models, since only limited data are available on the safety and immunogenicity of filovirus vaccines in humans. In the interest of conciseness, only vaccines that have been shown to be efficacious in NHP models are discussed. Rodent studies are included in order to describe correlates of immunity and testing of vaccine design and regimen development.

\section{Replicon-Based and Adenovirus-Based Vaccines}

Venezuelan equine encephalitis virus replicons. The Venezuelan equine encephalitis virus replicon particle (VRP) platform is based on an attenuated strain of Venezuelan equine encephalitis (VEE) virus replicon. ${ }^{30}$ Foreign genes can be inserted into the 
VEE replicon (replacing the VEE structural proteins), which is then transfected into cells together with helper constructs expressing VEE structural proteins. This generates VEE viruslike particles containing the replicon with the transgene of interest. The resultant VRP is a single-cycle, propagation-defective vector that generates large quantities of the foreign gene product within the cell it infects. The VRP platform has been used to express a wide variety of foreign genes.

VRPs expressing MARV GP protected guinea pigs from MARV infection after one, two or three vaccinations. ${ }^{31}$ Guinea pigs vaccinated with VRPs expressing only NP or VP35 were also protected from infection; however, VRPs expressing VP30 or VP24 were not protective. These results were used to formulate a vaccine regimen in NHPs. Cynomolgus macaques vaccinated three times with $10^{7}$ focus-forming units (FFU) VRP expressing MARV GP, NP or both, survived MARV challenge. ${ }^{31}$ All GP and GP/NP vaccinated animals survived without any evidence of sickness or viremia. Although two of three of NP vaccinated animals also survived, all three developed signs of MARV disease after infection. Anti-MARV antibody titers prior to challenge ranged from low to moderate, but neutralizing antibodies were not detected.

The VRP vaccine has been tested in mouse models to understand components required for protection and correlates of immunity. VRPs expressing EBOV GP, NP, VP24, VP30, VP35 or VP40 were shown to be protective in at least one mouse strain against EBOV infection. ${ }^{32-34}$ Passive transfer of sera from mice vaccinated with VRP-GP but not VRP-VP24, VP30, VP35 or $\mathrm{VP} 40$, protected recipient mice from EBOV infection. ${ }^{32}$ Adoptive transfer studies showed that $\mathrm{CD}^{+} \mathrm{T}$ cells, but not $\mathrm{CD}^{+} \mathrm{T}$ cells, from VRP-NP-vaccinated mice can protect naïve animals from EBOV challenge. ${ }^{33}$ Epitope mapping after vaccination revealed several peptides that induced $\mathrm{CD}^{+} \mathrm{T}$-cell cytokine production and cytotoxicity in $\mathrm{C} 57 \mathrm{BL} / 6$ and $\mathrm{BALB} / \mathrm{c}$ mice, establishing a useful system for consistent evaluation of T-cell responses after infection or vaccination. ${ }^{34}$

Additionally, guinea pigs were used to show that VRP and DNA vaccines against MARV were protective against aerosol challenge, an important consideration due to concerns over aerosol exposure. ${ }^{35}$ The VRP vaccine expressing GP or GP and NP, but not NP alone, was also effective against peripheral EBOV challenge in guinea pigs. ${ }^{36,37}$ Passive transfer of sera from VRPvaccinated mice or guinea pigs did not protect against EBOV infection. ${ }^{36}$

These studies illustrate the usefulness of the guinea pig and mouse models, as different VRP vaccines were evaluated in these animals, with the most successful ones later evaluated in NHPs. The VRP vaccine holds the distinction of being the first completely successful vaccine against a filovirus infection in NHPs. Additional work will hopefully be published testing the effectiveness of this vaccine platform in NHPs in different ebolavirus infections.

Adenovirus and DNA vaccines. Adenovirus serotype 5 (AdV) vectors can be used to express high levels of foreign transgenes in infected cells. AdV genes required for replication can be deleted and replaced with the transgene of interest. The AdV platform is non-replicating, and has been used in human gene therapy trials.

Initial studies. The first successful vaccine for protection against an ebolavirus in NHPs was based on a DNA-prime adenovirus-boost regimen. ${ }^{38}$ In this study, mice vaccinated with DNA encoding EBOV-GP and then boosted with AdV-EBOV GP generated higher antibody titers and stronger T-cell responses than mice vaccinated with DNA alone. Based on this data, cynomolgus macaques were vaccinated three times with DNA encoding EBOV NP and GP, then boosted three months later with $10^{10}$ particles of AdV vectors expressing EBOV GP. This regimen protected all NHPs from EBOV challenge. Three of the four vaccinated animals did not have viremia at any time point and the remaining animal had low viremia on day 10, which resolved by day 17. After DNA vaccination, moderate levels of antiEBOV antibodies were detected and titers greatly increased after the AdV-GP boost. In addition, peripheral blood mononuclear cells from vaccinated animals proliferated after incubation with EBOV GP in vitro. This proliferation was dependent on the presence of $\mathrm{CD}^{+}{ }^{+} \mathrm{T}$ cells, indicating the generation of T-cell memory.

Follow-up studies described a more rapid vaccination regimen. ${ }^{39}$ Mice vaccinated with AdV-EBOV-GP generated antibodies more rapidly than DNA-prime AdV-boosted mice. Therefore, cynomolgus macaques were vaccinated twice with $10^{12}$ particles of each of AdV-GP (EBOV) and AdV-NP (EBOV). Animals were challenged with EBOV one week after the second vaccination. All of the vaccinated animals survived challenge and none were viremic at any time point. Subsequent experiments found that only one vaccination with $10^{12}$ particles of each AdV-GP and $\mathrm{AdV}-\mathrm{NP}$ was protective against EBOV challenge and viremia was not detected in vaccinated animals at any point after EBOV infection. ${ }^{39}$ This one-shot vaccination did not generate detectable $\mathrm{CD}^{+}{ }^{+} \mathrm{T}$-cell memory responses (as measured by TNF $\alpha$ expression after stimulation with peptides spanning EBOV GP), but responses were detected six and ten days after challenge. CD8 ${ }^{+}$ T-cell responses were detected in five of eight animals either before or during infection. EBOV-specific antibody levels were low to moderate two weeks after single vaccination.

Dose requirements and vaccine modifications. Further experiments showed that lower doses of vaccine were protective against EBOV infection. A dose escalation study showed that a single vaccination with $10^{12}, 10^{11}$ or $10^{10}$ particles of each AdV-NP and AdV-GP protected cynomolgus macaques from EBOV challenge. ${ }^{40}$ However, animals receiving $10^{9}$ particles succumbed to infection. EBOV-specific $\mathrm{CD}^{+}{ }^{+} \mathrm{T}$-cell $\mathrm{TNF} \alpha$ responses prior to infection were strongest in animals receiving $10^{12}$ particles; however, no significant difference was detected in animals receiving $10^{10}$ versus $10^{9}$ particles. No difference in $\mathrm{CD}^{+} \mathrm{T}$-cell production of $\mathrm{TNF} \alpha$ was seen in any of the groups. Interestingly, however, there was a significant drop (roughly tenfold) in IgG titers between the $10^{10}$ particles group (which survived) compared to the $10^{9}$ particles group (which succumbed), suggesting that antibody levels, but not T-cell activity, correlated with protection in this study. Neutralizing antibody titers were low to undetectable in all groups. 
Additional development of the single-dose AdV platform focused on modifying the GP used in the vaccine preparation. ${ }^{40}$ Overexpression of EBOV GP in vitro can lead to cellular toxicity. ${ }^{41}$ Deletion of the transmembrane domain of EBOV GP, which eliminates the in vitro toxicity of GP, resulted in less protection against EBOV infection (compared to wild-type GP) when given with EBOV NP in the AdV vaccine platform. Surprisingly, CD $4^{+}$ and $\mathrm{CD}^{+}{ }^{+} \mathrm{T}$-cell responses and antibody titers were similar in these groups and neutralizing antibody was low or absent. In subsequent experiments, a GP containing a point mutation that reduced toxicity in vitro (GPE71D) was tested. ${ }^{40}$ Combination of AdV-GPE71D with AdV-NP (at $10^{12}$ particles each) protected two of three animals from EBOV challenge. However, AdVGPE71D (EBOV) and AdV-GPE71D (SUDV) at $10^{10}$ particles each protected macaques from EBOV challenge; interestingly, addition of AdV-NP (EBOV) to this vaccine preparation may have diminished efficacy, resulting in protection of only one of three animals. No differences in antibody titers or $\mathrm{CD}^{+}$or $\mathrm{CD}^{+}$T-cell responses were found among the groups.

Further studies utilized a complex adenovirus vector (CAdVax) that could carry larger or additional transgenes (compared to the previously used $\mathrm{AdV}$ vectors) to generate a vaccine that could protect against multiple filoviruses. Four vectors, each carrying one or two filovirus genes, were used together as a vaccine. ${ }^{42}$ The vaccine formulation consisted of the following genes: EBOV NP and GP, SUDV GP, MARV-Cieplik GP, RAVV GP and MARV-Musoke GP and NP. Cynomolgus macaques were vaccinated twice with $10^{10}$ particles of each of the four vectors. Vaccination induced antibody titers against all viruses, with high titers against EBOV and SUDV and moderate titers against MARV and RAVV. All vaccinated animals survived challenge with EBOV or MARV-Musoke and none developed viremia, fever or (with the exception of one animal) elevated AST or ALT serum levels. These animals were then challenged with MARVCieplik (EBOV survivors) or SUDV (MARV survivors) and all survived without showing signs of infection.

Subsequent studies used a single CAdVax vector carrying both SUDV GP and EBOV GP to vaccinate cynomolgus macaques. ${ }^{43}$ After two vaccinations with $10^{10}$ particles, these animals survived SUDV challenge and a subsequent EBOV challenge, without exhibiting any illness. A single vaccination was sufficient for protection against aerosolized EBOV, but two vaccinations were required for complete protection against aerosol SUDV challenge. Two rounds of vaccination with $10^{10}$ particles of two AdV vectors (SUDV GP/EBOV GP and MARV-Cieplik GP/RAVV GP) resulted in similar anti-EBOV antibody titers compared to the EBOV-only vaccine and also afforded protection against EBOV and SUDV challenge, suggesting inclusion of additional filovirus genes did not diminish immunogenicity.

One concern of using the AdV platform for vaccination is significant pre-existing immunity in humans to the adenovirus vector that has been used in most of the described studies. ${ }^{44}$ The mouse model was used to demonstrate that pre-existing immunity against $\mathrm{AdV}$ abrogated the ability of AdV-GP to protect from EBOV disease, ${ }^{45}$ although this could be overcome with vaccination via the intranasal route. ${ }^{46}$ Additionally, the use of a chimpanzee adenovirus pan 7 vector expressing EBOV GP was protective in laboratory mice and guinea pigs and immune responses to the vaccine were not affected by pre-existing immunity to the more common AdV-5 vector. ${ }^{45}$ Interestingly, studies in NHPs suggested that pre-existing anti-vector immunity did not abrogate the effectiveness of the standard AdV platform. Rhesus monkeys were vaccinated twice with $10^{9}$ particles of a CAdVax dengue virus vector to generate anti-AdV vector immunity. ${ }^{43}$ One year later, these animals were vaccinated twice with either $10^{10}, 10^{9}$ or $10^{8}$ particles of the SUDV-GP/EBOV-GP vaccine. Animals receiving the two highest doses of vaccine generated anti-EBOV antibodies at similar levels compared to vaccinated control animals that did not have pre-existing immunity against the AdV vector. However, animals receiving the lowest dose of vaccine had reduced titers. Nonetheless, all vaccinated animals survived aerosol EBOV challenge without developing any clinical signs of disease. Since it is usually assumed that mice are easier to protect against filovirus infection than NHPs, it is unclear why pre-existing anti-vector immunity against AdV abrogated AdV-GP vaccination in mice ${ }^{45}$ but not in NHPs. ${ }^{43}$ These studies suggest an important and often overlooked point-failure to protect with one vaccine or therapeutic regimen does not necessarily mean that the approach is ineffective. Alterations in design, dosage, route of administration or other factors can change the efficacy of a given vaccine.

The mouse model of EBOV infection was used to determine efficacy of routes of immunization and modified vectors for the $\mathrm{AdV}$ platform. Inoculation with AdV-GP via the intramuscular, nasal or oral routes protected mice from EBOV challenge. ${ }^{47}$ Splenic antigen-specific T-cell IFN $\gamma$ responses were highest in intramuscularly vaccinated animals, but responses in bronchiolar lavage, mesenteric lymph nodes and Peyer's patches were higher in nasally or orally vaccinated. The mouse model was also used to test an improved AdV-GP vector, containing a codon-optimized GP and enhanced promoter and translation sequences. ${ }^{48}$ This vector induced higher $\mathrm{T}$ - and $\mathrm{B}$-cell responses and was more protective at lower doses, than the standard AdV vector.

Comparison of DNA and adenovirus vaccination. Additional studies were performed to compare protection generated by DNA prime-AdV boost, DNA alone or AdV-only vaccination against MARV infection. ${ }^{49}$ Cynomolgus macaques were vaccinated four times with four milligrams of an improved DNA vaccine expressing MARV-Angola GP (DNA/DNA), once with $10^{11}$ particles of $\mathrm{AdV}$ expressing codon-optimized GP (AdV-only) or three times with DNA and boosted once with AdV-GP (DNA/AdV). After challenge with MARV-Angola, all vaccinated animals survived, regardless of vaccine regimen. AdV-only vaccinated animals did not develop disease signs following challenge, but mild to moderate signs were observed in some animals in the other two groups. Animals that exhibited symptoms had lower overall T-cell responses and lower polyfunctional $\mathrm{CD}^{+}{ }^{+} \mathrm{T}$-cell responses compared to asymptomatic animals in the same group. Antibody titers were similar in DNA/DNA versus AdV-only groups at the end of the vaccine regimen and were highest in the DNA/AdV animals. Little to no neutralizing antibody was detected in any group from this study. All vaccine regimens generated memory 
$\mathrm{CD}^{+}$and $\mathrm{CD}^{+}$T-cell responses, with the highest responders in the DNA/AdV group. DNA/AdV groups had a higher percentage of $\mathrm{CD}^{+}{ }^{+}$T-cells producing IFN $\gamma, \mathrm{TNF} \alpha$ and IL-2 upon stimulation with GP peptides when compared to those of AdVonly animals. Interestingly, polyfunctional CD8 ${ }^{+} \mathrm{T}$ cells (those producing two or three of the cytokines mentioned above) were most abundant in DNA/AdV groups, then DNA/DNA groups and lowest in $\mathrm{AdV}$-only groups, suggesting that measurement of polyfunctional $\mathrm{T}$ cells was not indicative of vaccine efficacy in this study. Interestingly, it previously had been reported that DNA vaccination against MARV GP was only partially protective in NHPs; ${ }^{50}$ the use of improved plasmids described above (codon-optimization and increased expression of GP) likely contributed to this more effective DNA vaccine.

Cross-protection against Bundibugyo virus. BDBV was discovered in 2007 and is more closely related to RESTV and TAFV than EBOV or SUDV. ${ }^{51}$ The DNA/AdV platform was used to test whether vaccination against EBOV and SUDV can crossprotect against BDBV infection. ${ }^{52}$ Cynomolgus macaques were vaccinated four times (at 4-6 wk intervals) with two milligrams each of plasmid DNA expressing EBOV GP and SUDV GP, followed by boosting with $10^{11}$ particles of $\mathrm{AdV}$ vaccine expressing EBOV GP one year after the final DNA vaccine. ${ }^{52}$ This regimen induced high antibody titers against EBOV GP after boosting, but no antibody titers against BDBV GP were detected. However, the vaccination did induce $\mathrm{CD}^{+}$and $\mathrm{CD}^{+}{ }^{+}$T-cell cytokine responses against both EBOV and BDBV GP. Remarkably, seven weeks after the AdV boost, all vaccinated animals survived challenged with BDBV, whereas only one of four control animals survived. Three of four vaccinated animals did not develop clinical signs after challenge, but one animal had mild symptoms and viremia that resolved. It was not reported if anti-BDBV antibodies were generated after challenge. It is important to note that one of the four the control animals survived, and that BDBV has the lowest reported lethality rate in humans $(-40 \%)$ of any ebolavirus (although based on very limited data), suggesting that BDBV may not be as pathogenic as other ebolaviruses. ${ }^{51}$ This is the first report of successful filovirus vaccination in NHPs without the appearance of specific antibody. This is surprising, since vaccination of rabbits with SUDV GP peptides generates cross-reactive antibodies against $\mathrm{EBOV},{ }^{53}$ and these are the most divergent of the ebolaviruses. Additionally, a mouse monoclonal antibody has been shown to bind EBOV, SUDV, RESTV and TAFV GP, ${ }^{53}$ suggesting that cross-reactive antibodies against ebolaviruses can readily be generated. Further research should be done to probe the requirement for antibody responses in combating BDBV infection.

Human trials. The first published human trial with a filovirus vaccine was a safety and efficacy DNA-only vaccine study against EBOV and SUDV. ${ }^{54}$ Twenty-seven human volunteers were vaccinated three times with either $0,2,4$ or $8 \mathrm{mg}$ of each of three plasmids, containing EBOV NP, EBOV GP and SUDV GP. The vaccine was well-tolerated and considered safe. All vaccinees generated antibodies to at least one of the proteins, and almost all developed antibodies against both GPs. Neutralizing antibody titers were not detected in any vaccinees. Antibody titers dropped to nearly undetectable levels one year after the last vaccination. $\mathrm{CD}^{+}{ }^{+} \mathrm{T}$-cell responses were generated in all subjects, with SUDV GP responses being strongest. Only about one quarter of vaccinees generated $\mathrm{CD}^{+} \mathrm{T}$-cell responses against the antigens.

Humans have also been vaccinated with two AdV vectors containing EBOV GP and SUDV GP.55 This Phase I study found that the vaccine was safe and well-tolerated. Patients received a single dose of $2 \times 10^{10}$ or $2 \times 10^{9}$ total vaccine particles. $\mathrm{CD}^{+}$or $\mathrm{CD}^{+}{ }^{+} \mathrm{T}$-cell responses were generated in less than half of vaccinated individuals. Antibody generation was dependent on dose, with roughly half of the patients vaccinated with the low-dose vaccine generating antibody against SUDV or EBOV, while all or roughly half of the high-dose group made anti-SUDV and anti-EBOV antibody, respectively. Patients having pre-existing antibodies against $\mathrm{AdV}$ had lower response rates and antibody titer magnitude compared to those who did not have pre-existing immunity against the vector. Interestingly, however, pre-existing immunity to $\mathrm{AdV}$ did not affect generation of memory $\mathrm{T}$ cells. In the NHP studies discussed earlier, antibody responses to similar vaccine doses were not diminished by pre-existing immunity against $\mathrm{AdV} ;{ }^{43}$ therefore, it appears that additional studies need to be performed to determine the impact of pre-existing antivector immunity on filovirus $\mathrm{AdV}$ vaccine efficacy.

The AdV platform has been well-tested in regards to dosing, vector modifications and comparison with or without a DNA priming vaccination. The importance of antibody titers as a correlate of immunity in EBOV infection has also been recorded for this platform. ${ }^{56}$ This is also the only filovirus vaccine platform in which polyfunctional T-cell responses in vaccinated NHPs have been investigated. Early clinical trials with either DNA or AdV vaccination have also been published. Further research on the impact of pre-existing immunity against the adenovirus vector on vaccine efficacy would be helpful; since natural infection of wild-type $\mathrm{AdV}$ may result in more robust anti-AdV immunity compared to vaccination with replication-deficient AdV (as done above), additional studies should be performed to address this issue.

\section{Live Virus Vaccines}

Vesicular stomatitis virus vaccines. Live attenuated vesicular stomatitis virus (VSV) vectors can be engineered to replace the VSV GP with a filovirus GP. The resulting vaccine platform can replicate in cells that filovirus GP targets in an attenuated manner.

Initial studies. The first published studies using VSV as a filovirus vaccine were conducted in mice, showing that the vaccine was protective against EBOV infection and replication of the vector was required for protection. ${ }^{57}$ Building on these results, VSV expressing GP from either EBOV or MARV were used to vaccinate NHPs against filovirus infection. ${ }^{58}$ Cynomolgus macaques were vaccinated once with $10^{7} \mathrm{pfu}$ of either vaccine and then challenged with EBOV or MARV. All vaccinated macaques that were challenged with homotypic virus survived infection, without any clinical signs or viremia. Immunized macaques developed low to moderate $\operatorname{IgG}$ titers by the day of challenge, but neutralizing antibody titers were low to undetectable. Interestingly, after 
challenge, IgG titers increased in three of four EBOV-vaccinated macaques by 28 days post-challenge, but IgG titers did not change after infection in VSV/MARV-GP-vaccinated animals. Filovirus-specific T-cell responses were not detected on the day of challenge in VSV/EBOV-vaccinated macaques. On day 6 postinfection, low levels of $\mathrm{CD}^{+}$T-cell responses were found, with somewhat higher responses seen on day 14. Interestingly, T-cell responses were not detected at any time point in VSV/MARVvaccinated animals after challenge. (Mouse studies revealed that $\mathrm{CD}^{+} \mathrm{T}$ cells were not required for VSV/EBOV-GP-mediated protection, and that adoptive transfer of sera from vaccinated animals protected recipient mice from EBOV infection ${ }^{26}$ ). To determine how the NHPs responded to the live VSV vaccine, the titers of VSV were analyzed after vaccination. ${ }^{58}$ The VSV/ EBOV-vaccinated macaques had low to moderate VSV viremia on day 2, but no viremia was detected at later time points. Four of six VSV/MARV-vaccinated macaques had low VSV viremia on day 2 after vaccination, with no detectable viremia after that time point. Similar VSV vaccination regimens protected NHPs against EBOV or MARV aerosol challenges. ${ }^{59}$ Additional work showed that a vaccine cocktail containing three VSV vectors (10 7 pfu each) expressing GP from either EBOV, SUDV or MARV protected cynomolgus macaques from EBOV, TAFV, SUDV or MARV challenge. ${ }^{60}$

Vaccination of cynomolgus macaques with $2 \times 10^{7} \mathrm{pfu}$ of VSV/EBOV-GP via the intramuscular, oral or intranasal routes protected animals from EBOV challenge. ${ }^{61}$ Intranasal vaccination induced increased $\operatorname{IgA} \operatorname{IgG}$ and $\operatorname{IgM}$ serum antibody titers compared to intramuscular vaccination. Oral vaccination also resulted in increased IgA titers pre-and post-challenge, IgG titers post-challenge and IgM titers pre-challenge, relative to intramuscular vaccination. High neutralizing antibody titers were generated in the orally vaccinated animals, moderate titers in the intranasal group and low titers in intramuscularly vaccinated animals. ELISPOT assays suggested that intramuscular vaccination generated stronger T-cell responses than oral or intranasal vaccination, but intracellular cytokine staining and proliferation studies suggested the opposite occurred after challenge.

Post-exposure vaccination. Rhesus monkeys were infected with MARV and treated with $10^{7}$ pfu VSV/MARV GP 20-30 min after infection. ${ }^{62}$ This treatment regimen protected all animals, with no detection of viremia or clinical signs of infection during the study time course. Control animals receiving VSV/EBOV and infected with MARV succumbed to infection as expected. Serological analysis revealed that low $\operatorname{IgM}$ responses against MARV had developed by day 6 after infection and generally subsided by day 14 . Low IgG titers were detected on day 10 , increased through day 14, and then leveled off. Neutralizing antibody titers also generally increased during the course of infection, but were still at low levels. Additionally, a T-cell cytokine response to MARV-GP was not detected at any point during infection.

This study was extended to MARV-infected rhesus monkeys given $2 \times 10^{7}$ pfu of VSV/MARV-GP vaccine 24 or $48 \mathrm{~h}$ after infection. ${ }^{63}$ Five of six animals survived in the 24-h treatment group, whereas two of six survived in the 48-h post-infection group. No viremia was detected in the surviving 24-h group of animals. This study suggests that extended post-exposure protection may be feasible with vaccination.

Similar post-exposure studies were also performed with EBOV. ${ }^{64}$ VSV/EBOV-GP protected mice from EBOV when given $30 \mathrm{~min}$ or $24 \mathrm{~h}$ after infection. Eighty-three percent and fifty percent of guinea pigs were protected from EBOV infection when given VSV/EBOV-GP one or 24 hours after infection, respectively. This work was continued in EBOV-infected rhesus monkeys. These animals were infected with EBOV and treated with $2 \times 10^{7}$ pfu of VSV/EBOV-GP 20-30 min after infection. ${ }^{64}$ This regimen protected four of eight macaques. The four surviving animals developed a range of clinical signs before resolving infection, but all had lower viremias on day 6 than control groups or the treated animals that died. Low IgM titers were detected in two of four surviving animals, whereas IgG was detected in all four surviving animals. Three of the four non-survivors did not develop antibodies. It is curious that $\operatorname{IgM}$ could not be detected in two of the four survivors, but that IgG was detected in these animals. Neutralizing antibodies were detected in all survivors but only in one of the four animals that succumbed.

A similar experiment with SUDV-infected rhesus monkeys resulted in four of four animals surviving after administration of VSV/SUDV-GP 20-30 min after infection. ${ }^{65}$ All treated animals displayed mild to moderate clinical signs, including fever, lymphopenia, viremia and elevation of liver enzymes. The control animal (infected but treated with VSV/Lassa virus GPC) died quite late, on day 17. All treated animals developed anti-SUDV IgM by day 6 and IgG by day 10, but the control surprisingly did not have any detectable antibodies, even on day 14.

Safety of vaccines in immunocompromised animals. Since there are currently no licensed live-virus-vectored vaccines approved by the FDA, safety is a concern for the VSV platform. Vaccination of mice lacking B and T cells (SCID mice) with VSV/EBOV-GP did not result in clinical manifestation of disease. ${ }^{26}$ To further address these concerns, investigators used VSV/EBOV-GP vectors in immunocompromised NHPs to assess safety of the vaccine. ${ }^{66}$ Simian/human immunodeficiency virus (SHIV)-infected rhesus monkeys were vaccinated with $10^{7}$ pfu of VSV/EBOV-GP. VSV viremia was detected on the second day after vaccination in four of six monkeys, and was not detected at any point afterwards. Four of the six vaccinated SHIV-infected monkeys survived EBOV virus challenge $31 \mathrm{~d}$ after vaccination, with no or only minor manifestations of disease. The two animals that succumbed to EBOV infection, however, were the two animals that had the lowest $\mathrm{CD} 4^{+}$T-cell counts. Additionally, these were the only two animals that had detectable SHIV viremia, suggesting that animals with active SHIV infection were not protected with the vaccine. Three of the four survivors did not have SHIV viremia during the vaccination course, while one had viremia on days 7 and 10 after vaccination but not at any later time point. Therefore, it is likely that the vaccine did not protect the two monkeys with active SHIV infection from EBOV infection, but did not have detectable adverse effects on these animals either.

Human studies. The VSV/EBOV-GP vaccine has been used in a human patient who had a needle-stick accident while working with EBOV. ${ }^{63}$ Approximately forty hours after the incident, the 
patient was injected with $\approx 5 \times 10^{7} \mathrm{pfu}$ of VSV/EBOV-GP. Hours after this vaccination, the patient experienced fever, myalgia and headache. The patient, fortunately, survived. Despite initial speculation that the vaccine protected against disease, ${ }^{67}$ it was later reported at a meeting that there was no evidence of actual infection; EBOV was never detected, nor was any antibody against any EBOV protein other than GP found. ${ }^{68}$

The VSV platform is the only filovirus vaccine that has been shown to have efficacy when given after infection. It is also an effective platform for a number of filoviruses. Additional work on deciphering correlates of immunity would be advantageous. Safety concerns over using a live-vectored vaccine in filoviral endemic regions, or in any population, remains; however, investigators have begun to address these concerns using immunodeficient mice and NHPs.

Human parainfluenzavirus 3 vaccines. Human parainfluenzavirus 3 (HPIV-3) is a common respiratory virus that targets cells in the respiratory tract. Filovirus genes can be inserted in the genome, and the resultant live replicating virus can be used as a vaccine.

A mucosal vaccine based on HPIV-3 has been tested against EBOV infection. Intranasal inoculation of guinea pigs with HPIV-3 expressing EBOV GP or NP completely protected against EBOV challenge. ${ }^{69}$ NHPs studies were then conducted to further assess the efficacy of this platform. Rhesus monkeys were immunized intranasally and intratracheally using several different regimens. ${ }^{70}$ First, animals were immunized with $4 \mathrm{x}$ $10^{6}$ TCID $_{50}$ of HPIV-3 carrying EBOV GP, EBOV GP and NP or EBOV GP and the cytokine GM-CSF (which can act as an adjuvant). Animals had low to moderate serum IgG titers, but serum IgA responses were undetectable. Both animals survived EBOV challenge in the HPIV-3-GP group, one of two survived in the HPIV-3-GP/NP group and two of two survived in the HPIV-3-GP/GM-CSF group. All survivors had no or only mild clinical signs after infection. In a subsequent experiment, one of two animals receiving $2 \times 10^{7}$ TCID $_{50}$ HPIV-3-GP died after EBOV infection and the survivor was sick before recovering, even though these animals had low to moderate titers of IgG and IgA. All animals receiving two doses of $2 \times 10^{7} \mathrm{TCID}_{50}$ of HPIV3-GP survived infection without signs of disease; these animals had low to moderate IgA titers and high IgG titers in sera after the second vaccination. Antigen-specific T-cell responses were low and variable after vaccinations.

To ameliorate the concern of pre-existing immunity against HPIV-3, an HPIV-3 mutant vector not expressing HPIV-3 surface molecules and expressing EBOV GP was used to vaccinate guinea pigs intranasally. ${ }^{71}$ This resulted in protection from EBOV challenge and suggested that such a vector might ameliorate concerns over pre-existing immunity against HPIV-3.

The HPIV-3 platform utilizes a common respiratory virus to vaccinate via the mucosal route. The advantage of this is the ability to generate mucosal immune responses, which may be important in a potential aerosol challenge. Concerns over pre-existing immunity and safety require additional studies, such as the above study modifying HPIV-3 surface proteins. Correlates of immunity and efficacy against other filoviruses are not known at this point.

\section{Protein-based Vaccines}

Virus-like particles. Filovirus-like particles (VLPs) are "empty shells" that have the morphology of filoviruses but lack the ability to replicate. ${ }^{72}$ Expression of a filovirus VP40 matrix protein in cells is necessary and sufficient to form a VLP. Addition of filovirus GP provides a target for neutralizing antibodies; other filoviral proteins, such as NP, can be incorporated into the VLP.

Initial studies showed that intramuscular or intraperitoneal vaccination with VLPs (containing VP40 and GP) protected mice from EBOV infection. ${ }^{22}$ VLPs also activated mouse dendritic cells in vitro and $\mathrm{T}$ and $\mathrm{B}$ cells in vivo. ${ }^{22}$ Vaccination of guinea pigs with EBOV VLPs resulted in antibody production and protection from EBOV challenge. ${ }^{73}$ In subsequent NHP studies, three rounds of vaccination of cynomolgus macaques with $250 \mu \mathrm{g}$ of EBOV VLPs containing VP40, GP, NP and RIBI adjuvant protected all animals from challenge with EBOV; viremia was not detected at any time point after challenge. ${ }^{74}$ Two of five macaques had a $2-3^{\circ} \mathrm{F}$ elevation of temperature on day 8 after challenge; liver enzyme or platelet levels were unchanged after challenge. Antibodies against EBOV were generated after a single vaccination and titers were boosted after a second vaccination. There was little difference in antibody titers between two and three vaccinations. Antibodies generated were capable of complement-mediated lysis and antibody-dependent cell-mediated cytotoxicity (ADCC) and low to moderate neutralizing titers were generated after vaccination. Additionally, strong T-cell responses (as measured by $\mathrm{TNF} \alpha$ production) were detected against EBOV GP or NP, but low responses against VP40 were found. T-cell responses against EBOV VP24 and VP35 were detected in vaccinated animals that survived challenge. Since these proteins were not part of the VLP preparation, this suggests a de novo T-cell response during infection, despite the lack of detectable viremia.

Prior to the development of a laboratory mouse model for MARV disease ${ }^{21}$ guinea pigs were the only rodent model for this virus. VLP vaccination induced antibodies and protected guinea pigs from MARV infection, ${ }^{75}$ and multiple vaccinations boosted antibody titers. Interestingly, $\mathrm{CD}^{+} \mathrm{T}$ cells, but not $\mathrm{CD}^{+}$ $\mathrm{T}$ cells, from vaccinated animals proliferated after stimulation with homotypic VLPs in vitro, suggesting the generation of $\mathrm{CD}^{+}{ }^{+}$T-cell immunity. ${ }^{76} \mathrm{~A}$ similar approach was taken to vaccinate cynomolgus macaques against MARV. ${ }^{76}$ Animals were vaccinated three times with $1 \mathrm{mg}$ of MARV-Musoke VLPs (containing VP40, NP and GP) and QS-21 adjuvant. All vaccinated animals survived challenge with either MARV-Musoke, MARVCieplik or RAVV and all had high antibody titers prior to challenge. No viremia was detected at any time after infection. Liver enzyme levels were also mostly unchanged, although one of the RAVV-challenged vaccinated macaques did have an increase in ALT concentrations, but did not develop viremia and survived.

A useful feature of VLPs is the ability to include proteins from different filoviruses in the same VLP. VP40 is sufficient to generate a VLP, but GP is often added to the VLP to induce generation of possible neutralizing antibodies and NP can be included as an additional target for T cells. This system was used to show 
that VLPs consisting of EBOV GP and MARV VP40 protected guinea pigs against EBOV, but not MARV, challenge. Similarly, VLPs made of MARV GP and EBOV VP40 were able to induce protective immune responses in guinea pigs against MARV, but not EBOV, infection. These data suggest that the GP is required for VLP-mediated protection in guinea pigs, although it is likely that the VP40-generated scaffold of VLPs is required for optimal induction of immune responses upon vaccination. Additional experiments showed that vaccination of guinea pigs with a cocktail of MARV and EBOV VLPs protected animals from challenge with either virus. ${ }^{73}$

The mouse model has been used to study immune correlates of protection in VLP-based vaccination against EBOV. Successful VLP vaccination required $\mathrm{CD} 8^{+} \mathrm{T}$ cells, $\mathrm{CD} 4^{+} \mathrm{T}$ cells, IFN $\gamma$ and $\mathrm{B}$ cells, but not perforin, for complete protection. ${ }^{24}$ Vaccination of mice with VLPs one to three days prior to infection also provided protection. ${ }^{25}$ In this scenario, NK cells and perforin were necessary for protection, but IFN $\gamma$ was not, suggesting that the timing of vaccination prior to EBOV infection may require different immune mechanisms to induce protection.

VLPs are non-replicating vaccines that do not have the safety concerns of other vectors. VLP vaccines against other pathogens, such as human papilloma virus, are approved for use in humans. Correlates of immunity in filoviral VLP vaccines are fairly welldefined. Dosing regimen, vaccine component requirements and time-to-immunity should be topics for future experimentation.

\section{Correlates of Immunity}

Immune responses to filovirus vaccination have been analyzed using a variety of assays. Antibody titers are measured by ELISA, using whole filovirus antigen (or purified GP) as a target. Neutralizing antibody titers are determined by the ability of serum to inhibit viral plaque formation (or plaque size, depending on the assay). T-cell responses are typically more difficult to measure. These assays measure antigen-specific cytokine production (or cellular proliferation) to overlapping filovirus peptides or whole antigen. Lastly, in vitro experiments analyze cytokine production and/or functional activity of purified cell types in response to the vaccine itself. Different studies tend to use different modifications of these assays, making direct comparisons difficult. A survey of the literature in this area is summarized below.

Antibody. The role of antibody in vaccine-mediated protection against filoviruses is not well-defined. It is difficult to compare antibody levels from one study to another, since different groups use different methods to report titers. For example, some studies define a positive signal as anything above a set O.D., whereas other groups report titers of vaccinated animals normalized to sera from unvaccinated animals. Additionally, some researches use whole irradiated virus as a target, while others use purified GP. Therefore, comparing antibody titers across studies is usually not an exact science. Regardless, the vaccines discussed in this review generate a broad range of filovirus specific antibody titers in NHPs, ranging from undetectable to high. Protection from infection was found all across this range. For instance, antibodies against BDBV were not found after vaccination with
DNA/AdV regimen against SUDV and EBOV, but the animals were protected from BDBV challenge. ${ }^{22}$ However, studies with $\mathrm{AdV}$ vectors expressing modified EBOV GP found a correlation between antibody titers and protection against $\mathrm{EBOV}$ challenge. ${ }^{40}$ Additionally, it has been shown that in various $\mathrm{AdV}$ vectored filovirus vaccines, all NHPs that had antibody titers above a certain level survived EBOV challenge; in animals that had antibody titers below this level there was a mixture of survival and death. ${ }^{56}$ Therefore, it appears that antibody levels required for protection must be determined for each individual vaccine platform.

Neutralizing antibody levels were low to undetectable in most vaccines reported here. However, the definition of "neutralizing" is often not clear. This nomenclature does not necessarily differentiate between elimination of plaque formation versus a decrease in plaque size. Therefore, further research must be performed to clarify the importance of neutralizing antibodies in vaccination against filoviruses. However, the current data suggests that high levels of neutralizing antibody are not required for protection against filovirus disease. This may be similar to certain arenavirus infections, where neutralizing antibody is not generated in patients surviving an initial infection, although neutralizing antibodies are required for protection after passive transfer of immune sera.

It is important to note that measurement of total antibody titers or neutralizing titers does not necessarily correlate with functional activity, since antibodies can act in a number of ways in vivo (ADCC, complement fixation, opsonization, etc.). Additionally, it is possible that antibodies may be generated against non-protective epitopes. Rodent studies have shown that passive transfer of immune sera is protective in some vaccine platforms but not in others. However, in any adoptive or passive transfer study it must be noted that results may be affected depending on dosage, potency of the transferred material and timing of administration. The subtype of antibodies generated by vaccination is an important, but often overlooked, parameter. VLP vaccination against $E B O V$ in mice induced all subtypes of IgG, with $I_{g G}>I g G_{2 b}>I g G_{2 a}>I g G_{3}$. Vaccination with VSV-GP, however, induced mainly $\operatorname{IgG}_{2 a}$, with negligible levels of other IgG subtypes. Therefore, additional in vivo studies must be performed to test the functionality of antibodies before definitive conclusions can be reached on effectiveness.

$T$ cells. T-cell responses in vaccinated NHPs also differ depending on the vaccine used. VLPs appear to generate strong T-cell responses in NHPs, while VSV-based vaccines do not. ${ }^{58,74,76}$ Studies in the EBOV mouse model confirm this finding, since $\mathrm{CD}^{+} \mathrm{T}$ cells are required for VLP but not VSVmediated protection. ${ }^{24,26}$ NHPs vaccinated with EBOV and SUDV GP via a DNA/AdV prime-boost regimen generated T cell, but not antibody, responses against BDBV. ${ }^{52}$ These animals were protected against BDBV challenge, suggesting that T-cell responses are necessary for protection, whereas antibody levels (at least prior to challenge) are not indicative of successful vaccination in this case. ${ }^{52}$ In agreement with this finding, stronger T-cell responses were roughly associated with decrease in clinical signs of AdV-platform vaccinated, MARV-challenged NHPs. ${ }^{49}$ On the other hand, AdV vaccines expressing modified EBOV GP 
(lacking the transmembrane domain) were less protective than those carrying wild-type GP, but there was no difference in measured T-cell responses between these groups. ${ }^{40}$ In post-exposure VSV vaccination against MARV in NHPs, however, T-cell responses were not detected at any time point. ${ }^{62}$ Additionally, T-cell responses were not detected against EBOV or MARV prior to challenge in VSV-vaccinated NHPs. ${ }^{58}$

Unfortunately, many of the published filovirus studies do not show representative flow plots along with bar graphs summarizing T-cell cytokine responses; this primary data is vital to display the quality of data generated with this difficult assay. It should be noted that there are many different ways to analyze T-cell function. Epitope-specific production of different cytokines such as TNF $\alpha$, IFN $\gamma$, IL- 2 and MIP-1 $\beta$ can provide some information on the possible in vivo function and potency of T cells. Other parameters, such as proliferative capacity and cytotoxicity, also can be informative about T-cell activity. Therefore, a more thorough examination of ex vivo B- and T-cell responses, as well as innate immune cell activity, is needed before conclusions can be reached on correlates of immunity for each vaccine platform and filovirus.

Other immune components. VLP studies have utilized knockout mice and in vitro assays to delineate correlates of immunity against EBOV. IFN $\gamma$, but not perforin, are required for protection against EBOV infection in VLP-vaccinated mice. ${ }^{24}$ However, successful rapid vaccination (1-3 d before infection) required $\mathrm{NK}$ cells, which used perforin but not IFN $\gamma$, to induce protection, ${ }^{25}$ suggesting that mechanisms of immunity may vary depending on time of administration of the vaccine. In vitro studies demonstrated that EBOV VLPs activate human NK cells, resulting in increased cytokine production and cytotoxicity. ${ }^{77}$ EBOV VLP-activated human NK cells also acquired the ability to kill EBOV infected dendritic cells via perforin and FasL-mediated cytotoxicity and this activity was at least partially dependent on the natural cytotoxicity receptor NKp30. VLPs also activate human dendritic cells in vitro. ${ }^{78}$

\section{References}

1. Kuhn JH. Filoviruses. A compendium of 40 years of epidemiological, clinical and laboratory studies. Arch Virol 2008; 20:13-360.

2. Warfield KL, Deal EM, Bavari S. Filovirus infections. J Am Vet Med Assoc 2009; 234:1130-9.

3. Kuhn JH, Becker S, Ebihara H, Geisbert TW, Johnson KM, Kawaoka Y, et al. Proposal for a revised taxonomy of the family Filoviridae: classification, names of taxa and viruses and virus abbreviations. Arch Virol 2010; 155:2083-103.

4. Jaax N, Jahrling P, Geisbert T, Geisbert J, Steele K, McKee K, et al. Transmission of Ebola virus (Zaire strain) to uninfected control monkeys in a biocontainment laboratory. Lancet 1995; 346:1669-71.

5. Johnson E, Jaax N, White J, Jahrling P. Lethal experimental infections of rhesus monkeys by aerosolized Ebola virus. Int J Exp Pathol 1995; 76:227-36.

6. Dalgard DW, Hardy RJ, Pearson SL, Pucak GJ, Quander RV, Zack PM, et al. Combined simian hemorrhagic fever and Ebola virus infection in cynomolgus monkeys. Lab Anim Sci 1992; 42:152-7.

7. 2004. Category A agents. In Centers for Disease Control and Prevention; http://www.bt.cdc.gov/Agent/ agentlist.asp.

8. Towner JS, Pourrut X, Albarino CG, Nkogue CN, Bird $\mathrm{BH}$, Grard G, et al. Marburg virus infection detected in a common African bat. PLoS One 2007; 2:764.
9. Pourrut X, Souris M, Towner JS, Rollin PE, Nichol ST, Gonzalez JP, Leroy E. Large serological survey showing cocirculation of Ebola and Marburg viruses in Gabonese bat populations and a high seroprevalence of both viruses in Rousettus aegyptiacus. BMC Infect Dis 2009; 9:159.

10. Leroy EM, Epelboin A, Mondonge V, Pourrut X, Gonzalez JP, Muyembe-Tamfum JJ, Formenty P. Human Ebola outbreak resulting from direct exposure to fruit bats in Luebo, Democratic Republic of Congo 2007. Vector Borne Zoonotic Dis 2009; 9:723-8.

11. Pourrut X, Delicat A, Rollin PE, Ksiazek TG, Gonzalez JP, Leroy EM. Spatial and temporal patterns of Zaire ebolavirus antibody prevalence in the possible reservoir bat species. J Infect Dis 2007; 196:176-83.

12. Barrette RW, Metwally SA, Rowland JM, Xu L, Zaki SR, Nichol ST, et al. McIntosh. Discovery of swine as a host for the Reston ebolavirus. Science 2009; 325:204-6.

13. Riabchikova EI, Kolesnikova LV, Rassadkin Iu N. [Microscopic study of species specific features of hemostatic impairment in Ebola virus infected monkeys]. Vestn Ross Akad Med Nauk 1998; 51-5.

14. Baskerville A, Bowen ET, Platt GS, McArdell LB, Simpson DI. The pathology of experimental Ebola virus infection in monkeys. J Pathol 1978; 125:131-8.

15. Bowen ET, Platt GS, Simpson DI, McArdell LB, Raymond RT. Ebola haemorrhagic fever: experimental infection of monkeys. Trans R Soc Trop Med Hyg 1978; 72:188-91.

\section{Conclusions}

Several vaccines have resulted in protection of NHPs against the data that are currently available, types of immunity. Antibody depletion studies of immune com ponents in NHPs would shed more light on how these vaccines k to generate protective immunity. There is a great need for of human and NHP filovirus studies. There are throughout the filovirus community, but have not been published or peer-reviewed. Publication of such data will only add to knowledge concerning filovirus infection, diagnosis We thank Jens Kuhn, Amy Shurtleff and Gordon Ruthel for part by the Joint Science and Technology Office program project number 1.1C0003_08_RD_B from the Department of Defense Chemical and Biological Defense program through the Defense Threat Reduction Agency (DTRA) (S.B.); and in part by an appointment to the Postgraduate Research Participation Program at the US Army Medical Research Institute for Infectious US Department of Er Materiel Command (S.B.B.).

The content of this publication does not necessarily reflect the views or policies of the US Department of Defense or the US Department of the Army.

16. Ryabchikova EI, Kolesnikova LV, Luchko SV. An analysis of features of pathogenesis in two animal models of Ebola virus infection. J Infect Dis 1999; 179:199-202.

17. Siegert R, Shu HL, Slenczka HL, Peters D, Muller G. The aetiology of an unknown human infection transmitted by monkeys (preliminary communication). Ger Med Mon 1968; 13:1-2.

18. Bray M, Davis K, Geisbert T, Schmaljohn C, Huggins J. A mouse model for evaluation of prophylaxis and therapy of Ebola hemorrhagic fever. J Infect Dis 1998; 178:651-61.

19. Connolly BM, Steele KE, Davis KJ, Geisbert TW, Kell WM, Jaax NK, Jahrling PB. Pathogenesis of experimental Ebola virus infection in guinea pigs. J Infect Dis 1999; 179:203-17.

20. Hevey M, Negley D, Geisbert J, Jahrling P, Schmaljohn A. Antigenicity and vaccine potential of Marburg virus glycoprotein expressed by baculovirus recombinants. Virology 1997; 239:206-16.

21. Warfield KL, Bradfute SB, Wells J, Lofts L, Cooper MT, Alves DA, et al. Development and characterization of a mouse model for Marburg hemorrhagic fever. J Virol 2009; 83:6404-15.

22. Warfield KL, Bosio CM, Welcher BC, Deal EM, Mohamadzadeh M, Schmaljohn A, et al. Ebola viruslike particles protect from lethal Ebola virus infection. Proc Natl Acad Sci USA 2003; 100:15889-94. 
23. Warfield KL, Posten NA, Swenson DL, Olinger GG, Esposito D, Gillette WK, et al. Filovirus-like particles produced in insect cells: immunogenicity and protection in rodents. J Infect Dis 2007; 196:421-9.

24. Warfield KL, Olinger G, Deal EM, Swenson DL, Bailey M, Negley DL, et al. Induction of humoral and $\mathrm{CD}^{+} \mathrm{T}$ cell responses are required for protection against lethal Ebola virus infection. J Immunol 2005; 175:1184-91.

25. Warfield KL, Perkins JG, Swenson DL, Deal EM, Bosio CM, Aman MJ, et al. Role of natural killer cells in innate protection against lethal ebola virus infection. J Exp Med 2004; 200:169-79.

26. Jones SM, Stroher U, Fernando L, Qiu X, Alimonti J, Melito P, et al. Assessment of a vesicular stomatitis virus-based vaccine by use of the mouse model of Ebola virus hemorrhagic fever. J Infect Dis 2007; 196:404-12.

27. Mikhailov VV, Borisevich IV, Chernikova NK, Potryvaeva NV, Krasnianskii VP. [The evaluation in hamadryas baboons of the possibility for the specific prevention of Ebola fever]. Vopr Virusol 1994; 39:82-4.

28. Chupurnov AA, Chernukhin IV, Ternovoi VA, Kudoiarova NM, Makhova NM, Azaev M, Smolina MP. [Attempts to develop a vaccine against Ebola fever]. Vopr Virusol 1995; 40:257-60.

29. Ignatyev GM, Agafonov AP, Streltsova MA, Kashentseva EA. Inactivated Marburg virus elicits a nonprotective immune response in Rhesus monkeys. J Biotechnol 1996; 44:111-8.

30. Pushko P, Parker M, Ludwig GV, Davis NL, Johnston RE, Smith JF. Replicon-helper systems from attenuated Venezuelan equine encephalitis virus: expression of heterologous genes in vitro and immunization against heterologous pathogens in vivo. Virology 1997; 239:389-401.

31. Hevey M, Negley D, Pushko P, Smith J, Schmaljohn A. Marburg virus vaccines based upon alphavirus replicons protect guinea pigs and nonhuman primates. Virology 1998; 251:28-37.

32. Wilson JA, Bray M, Bakken R, Hart MK. Vaccine potential of Ebola virus VP24, VP30, VP35 and VP40 proteins. Virology 2001; 286:384-90.

33. Wilson JA, Hart MK. Protection from Ebola virus mediated by cytotoxic $T$ lymphocytes specific for the viral nucleoprotein. J Virol 2001; 75:2660-4.

34. Olinger GG, Bailey MA, Dye JM, Bakken R, Kuehne A, Kondig J, et al.. Protective cytotoxic T-cell responses induced by venezuelan equine encephalitis virus replicons expressing Ebola virus proteins. J Virol 2005; 79:14189-96.

35. Hevey M, Negley D, VanderZanden L, Tammariello RF, Geisbert J, Schmaljohn C, et al. Marburg virus vaccines: comparing classical and new approaches. Vaccine 2001; 20:586-93.

36. Pushko P, Bray M, Ludwig GV, Parker M, Schmaljohn A, Sanchez A, et al. Recombinant RNA replicons derived from attenuated Venezuelan equine encephalitis virus protect guinea pigs and mice from Ebola hemorrhagic fever virus. Vaccine 2000; 19:142-53.

37. Pushko P, Geisbert J, Parker M, Jahrling P, Smith J. Individual and bivalent vaccines based on alphavirus replicons protect guinea pigs against infection with Lassa and Ebola viruses. J Virol 2001; 75:11677-85.

38. Sullivan NJ, Sanchez A, Rollin PE, Yang ZY, Nabel GJ. Development of a preventive vaccine for Ebola virus infection in primates. Nature 2000; 408:605-9.

39. Sullivan NJ, Geisbert TW, Geisbert JB, Xu L, Yang ZY, Roederer M, et al. Accelerated vaccination for Ebola virus haemorrhagic fever in non-human primates. Nature 2003; 424:681-4.

40. Sullivan NJ, Geisbert TW, Geisbert JB, Shedlock DJ, Xu L, Lamoreaux L, et al. Immune protection of nonhuman primates against Ebola virus with single low-dose adenovirus vectors encoding modified GPs. PLoS Med 2006; 3:177.
41. Yang ZY, Duckers HJ, Sullivan NJ, Sanchez A, Nabel EG, Nabel GJ. Identification of the Ebola virus glycoprotein as the main viral determinant of vascular cell cytotoxicity and injury. Nat Med 2000; 6:886-9.

42. Swenson DL, Wang D, Luo M, Warfield KL, Woraratanadharm J, Holman DH, et al. Vaccine to confer to nonhuman primates complete protection against multistrain Ebola and Marburg virus infections. Clin Vaccine Immunol 2008; 15:460-7.

43. Pratt WD, Wang D, Nichols DK, Luo M, Woraratanadharm J, Dye JM, et al. Protection of nonhuman primates against two species of Ebola virus infection with a single complex adenovirus vector. Clin Vaccine Immunol 2010; 17:572-81.

44. Nwanegbo E, Vardas E, Gao W, Whittle H, Sun H, Rowe D, et al. Prevalence of neutralizing antibodies to adenoviral serotypes 5 and 35 in the adult populations of The Gambia, South Africa, and the United States. Clin Diagn Lab Immunol 2004; 11:351-7.

45. Kobinger GP, Feldmann H, Zhi Y, Schumer G, Gao G, Feldmann F, et al. Chimpanzee adenovirus vaccine protects against Zaire Ebola virus. Virology 2006; 346:394-401

46. Croyle MA, Patel A, Tran KN, Gray M, Zhang Y, Strong JE, et al. Nasal delivery of an adenovirus-based vaccine bypasses pre-existing immunity to the vaccine carrier and improves the immune response in mice. PLoS One 2008; 3:3548.

47. Patel A, Zhang Y, Croyle M, Tran K, Gray M, Strong $\mathrm{J}$, et al. Mucosal delivery of adenovirus-based vaccine protects against Ebola virus infection in mice. J Infect Dis 2007; 196:413-20.

48. Richardson JS, Yao MK, Tran KN, Croyle MA, Strong JE, Feldmann H, Kobinger GP. Enhanced protection against Ebola virus mediated by an improved adenovirus-based vaccine. PLoS One 2009; 4:5308.

49. Geisbert TW, Bailey M, Geisbert JB, Asiedu C Roederer M, Grazia-Pau M, et al. Vector choice determines immunogenicity and potency of genetic vaccines against Angola Marburg virus in nonhuman primates. J Virol 2010; 84:10386-94

50. Riemenschneider J, Garrison A, Geisbert J, Jahrling P, Hevey M, Negley D, et al. Comparison of individual and combination DNA vaccines for B. anthracis, Ebola virus, Marburg virus and Venezuelan equine encephalitis virus. Vaccine 2003; 21:4071-80.

51. Towner JS, Sealy TK, Khristova ML, Albarino CG, Conlan S, Reeder SA, et al. Newly discovered ebol virus associated with hemorrhagic fever outbreak in Uganda. PLoS Pathog 2008; 4:1000212.

52. Hensley LE, Mulangu S, Asiedu C, Johnson J, Honko AN, Stanley D, et al. Demonstration of cross-protective vaccine immunity against an emerging pathogenic Ebolavirus Species. PLoS Pathog 2010; 6:1000904.

53. Yu JS, Liao HX, Gerdon AE, Huffman B, Scearce RM, McAdams M, et al. Detection of Ebola virus envelope using monoclonal and polyclonal antibodies in ELISA, surface plasmon resonance and a quartz crysta microbalance immunosensor. J Virol Methods 2006; 137:219-28.

54. Martin JE, Sullivan NJ, Enama ME, Gordon IJ, Roederer M, Koup RA, et al. A DNA vaccine for Ebola virus is safe and immunogenic in a phase I clinical trial. Clin Vaccine Immunol 2006; 13:1267-77.

55. Ledgerwood JE, Costner P, Desai N, Holman L, Enama ME, Yamshchikov G, et al. A replication defective recombinant Ad5 vaccine expressing Ebola virus GP is safe and immunogenic in healthy adults. Vaccine 2010; 29:304-13.

56. Sullivan NJ, Martin JE, Graham BS, Nabel GJ Correlates of protective immunity for Ebola vaccines: implications for regulatory approval by the animal rule. Nat Rev Microbiol 2009; 7:393-400.
57. Garbutt M, Liebscher R, Wahl-Jensen V, Jones S, Moller P, Wagner R, et al. Properties of replicationcompetent vesicular stomatitis virus vectors expressing glycoproteins of filoviruses and arenaviruses. J Virol 2004; 78:5458-65.

58. Jones SM, Feldmann H, Stroher U, Geisbert JB, Fernando L, Grolla A, et al. Live attenuated recombinant vaccine protects nonhuman primates against Ebola and Marburg viruses. Nat Med 2005; 11:786-90.

59. Geisbert TW, Daddario-Dicaprio KM, Geisbert JB, Reed DS, Feldmann F, Grolla A, et al. Vesicular stomatitis virus-based vaccines protect nonhuman primates against aerosol challenge with Ebola and Marburg viruses. Vaccine 2008; 26:6894-900.

60. Geisbert TW, Geisbert JB, Leung A, DaddarioDiCaprio KM, Hensley LE, Grolla A, Feldmann H. Single-injection vaccine protects nonhuman primates against infection with marburg virus and three species of ebola virus. J Virol 2009; 83:7296-304.

61. Qiu X, Fernando L, Alimonti JB, Melito PL, Feldmann F, Dick D, et al. Mucosal immunization of cynomolgus macaques with the VSVDeltaG/ZEBOVGP vaccine stimulates strong ebola GP-specific immune responses. PLoS One 2009; 4:5547.

62. Daddario-DiCaprio KM, Geisbert TW, Stroher U, Geisbert JB, Grolla A, Fritz EA, et al. Postexposure protection against Marburg haemorrhagic fever with recombinant vesicular stomatitis virus vectors in nonhuman primates: an efficacy assessment. Lancet 2006; 367:1399-404.

63. Geisbert TW, Hensley LE, Geisbert JB, Leung A, Johnson JC, Grolla A, Feldmann H. Postexposure treatment of Marburg virus infection. Emerg Infect Dis 2010; 16:1119-22.

64. Feldmann H, Jones SM, Daddario-DiCaprio KM, Geisbert JB, Stroher U, Grolla A, et al. Effective postexposure treatment of Ebola infection. PLoS Pathog 2007; 3:2.

65. Geisbert TW, Daddario-DiCaprio KM, Williams KJ, Geisbert JB, Leung A, Feldmann F, et al. Recombinant vesicular stomatitis virus vector mediates postexposure protection against Sudan Ebola hemorrhagic fever in nonhuman primates. J Virol 2008; 82:5664-8.

66. Geisbert TW, Daddario-Dicaprio KM, Lewis MG Geisbert JB, Grolla A, Leung A, et al. Vesicular stomatitis virus-based ebola vaccine is well-tolerated and protects immunocompromised nonhuman primates. PLoS Pathog 2008; 4:1000225.

67. Tuffs A. Experimental vaccine may have saved Hamburg scientist from Ebola fever. BMJ 2009; 338:1223.

68. Feldmann H. Replication-competent vaccines for filoviruses. In $5^{\text {th }}$ International Symposium on Filoviruses. Tokyo, Japan 2010.

69. Bukreyev A, Yang L, Zaki SR, Shieh WJ, Rollin PE, Murphy BR, et al. A single intranasal inoculation with a paramyxovirus-vectored vaccine protects guinea pigs against a lethal-dose Ebola virus challenge. J Virol 2006; 80:2267-79.

70. Bukreyev A, Rollin PE, Tate MK, Yang L, Zaki SR, Shieh WJ, et al. Successful topical respiratory tract immunization of primates against Ebola virus. J Virol 2007; 81:6379-88.

71. Bukreyev A, Marzi A, Feldmann F, Zhang L, Yang L, Ward JM, et al. Chimeric human parainfluenza virus bearing the Ebola virus glycoprotein as the sole surface protein is immunogenic and highly protective against Ebola virus challenge. Virology 2009; 383:348-61.

72. Bavari S, Bosio CM, Wiegand E, Ruthel G, Will AB, Geisbert TW, et al. Lipid raft microdomains: a gateway for compartmentalized trafficking of Ebola and Marburg viruses. J Exp Med 2002; 195:593-602.

73. Swenson DL, Warfield KL, Negley DL, Schmaljohn A, Aman MJ, Bavari S. Virus-like particles exhibit potential as a pan-filovirus vaccine for both Ebola and Marburg viral infections. Vaccine 2005; 23:3033-42. 
74. Warfield KL, Swenson DL, Olinger GG, Kalina WV, Aman MJ, Bavari S. Ebola virus-like particle-based vaccine protects nonhuman primates against lethal Ebola virus challenge. J Infect Dis 2007; 196:430-7.

75. Warfield KL, Swenson DL, Negley DL, Schmaljohn AL, Aman MJ, Bavari S. Marburg virus-like particles protect guinea pigs from lethal Marburg virus infection. Vaccine 2004; 22:3495-502.

76. Swenson DL, Warfield KL, Larsen T, Alves DA, Coberley SS, Bavari S. Monovalent virus-like particle vaccine protects guinea pigs and nonhuman primates against infection with multiple Marburg viruses. Expert Rev Vaccines 2008; 7:417-29.

77. Fuller CL, Ruthel G, Warfield KL, Swenson DL, Bosio CM, Aman MJ, Bavari S. NKp30-dependent cytolysis of filovirus-infected human dendritic cells. Cell Microbiol 2007; 9:962-76.

78. Bosio CM, Moore BD, Warfield KL, Ruthel G, Mohamadzadeh M, Aman MJ, Bavari S. Ebola and Marburg virus-like particles activate human myeloid dendritic cells. Virology 2004; 326:280-7.

79. Pattyn S, van der Groen G, Courteille G, Jacob W, Piot P. Isolation of Marburg-like virus from a case of haemorrhagic fever in Zaire. Lancet 1977; 1:573-4.

80. Moe JB, Lambert RD, Lupton HW. Plaque assay for Ebola virus. J Clin Microbiol 1981; 13:791-3.

81. van der Groen G, Jacob W, Pattyn SR. Ebola virus virulence for newborn mice. J Med Virol 1979; 4:239-40.
82. Bowen ET, Lloyd G, Harris WJ, Platt GS, Baskerville A, Vella EE. Viral haemorrhagic fever in Southern Sudan and northern Zaire. Preliminary studies on the aetiological agent. Lancet 1977; 1:571-3.

83. Bray M. The role of the Type I interferon response in the resistance of mice to filovirus infection. J Gen Virol 2001; 82:1365-73.

84. Fisher-Hoch SP, Brammer TL, Trappier SG, Hutwagner LC, Farrar BB, Ruo SL, et al. Pathogenic potential of filoviruses: role of geographic origin of primate host and virus strain. J Infect Dis 1992; 166:753-63.

85. Bowen ET, Platt GS, Lloyd G, Raymond RT, Simpson DI. A comparative study of strains of Ebola virus isolated from Southern Sudan and northern Zaire in 1976. J Med Virol 1980; 6:129-38

86. 1978. Ebola haemorrhagic fever in Sudan. Report of a WHO/International Study Team. Bull World Health Organ 1976; 56:247-70.

87. Ksiazek TG, West CP, Rollin PE, Jahrling PB, Peters CJ. ELISA for the detection of antibodies to Ebola viruses. J Infect Dis 1999; 179:192-8.

88. Jahrling PB, Geisbert TW, Dalgard DW, Johnson ED Ksiazek TG, Hall WC, Peters CJ. Preliminary report: isolation of Ebola virus from monkeys imported to USA. Lancet 1990; 335:502-5.

89. Hofmann H, Kunz C. [A strain of "Marburg virus" (Rhabdovirus simiae) pathogenic to mice]. Arch Gesamte Virusforsch 1970; 32:244-8.
90. Warfield KL, Alves DA, Bradfute SB, Reed DK Vantongeren S, Kalina WV, et al. Development of a model for marburgvirus based on severe-combined immunodeficiency mice. Virol J 2007; 4:108.

91. Simpson DI. Marburg agent disease: in monkeys. Trans R Soc Trop Med Hyg 1969; 63:303-9.

92. Johnson ED, Johnson BK, Silverstein D, Tukei P, Geisbert TW, Sanchez AN, Jahrling PB. Characterization of a new Marburg virus isolated from a 1987 fatal case in Kenya. Arch Virol 1996; 11:101-14.

93. Daddario-DiCaprio KM, Geisbert TW, Geisber JB, Stroher U, Hensley LE, Grolla A, et al. Crossprotection against Marburg virus strains by using a live, attenuated recombinant vaccine. J Virol 2006; 80:9659-66.

94. Wang D, Hevey M, Juompan LY, Trubey CM, Raja NU, Deitz SB, et al. Complex adenovirus-vectored vaccine protects guinea pigs from three strains of Marburg virus challenges. Virology 2006; 353:324-32.

95. Wang D, Raja NU, Trubey CM, Juompan LY, Luo M, Woraratanadharm J, et al. Development of a cAdVaxbased bivalent ebola virus vaccine that induces immune responses against both the Sudan and Zaire species of Ebola virus. J Virol 2006; 80:2738-46.

96. Warfield KL, Swenson DL, Demmin G, Bavari S Filovirus-like particles as vaccines and discovery tools. Expert Rev Vaccines 2005; 4:429-40. 